\title{
Defending Continuous Variable Teleportation: Why a laser is a clock, not a quantum channel.
}

\author{
Howard M. Wiseman \\ Centre for Quantum Computer Technology, Centre for Quantum Dynamics, \\ School of Science, Griffith University, Brisbane, Queensland 4111 Australia \\ E-mail: H.Wiseman@griffith.edu.au
}

\begin{abstract}
It has been argued [T. Rudolph and B.C. Sanders, Phys. Rev. Lett. 87, 077903 (2001)] that continuous-variable quantum teleportation at optical frequencies has not been achieved because the source used (a laser) was not 'truly coherent'. Van Enk, and Fuchs [Phys. Rev. Lett, 88, 027902 (2002)], while arguing against Rudolph and Sanders, also accept that an 'absolute phase' is achievable, even if it has not been achieved yet. I will argue to the contrary that 'true coherence' or 'absolute phase' is always illusory, as the concept of absolute time (at least for frequencies beyond direct human experience) is meaningless. All we can ever do is to use an agreed time standard. In this context, a laser beam is fundamentally as good a 'clock' as any other. I explain in detail why this claim is true, and defend my argument against various objections. In the process I discuss super-selection rules, quantum channels, and the ultimate limits to the performance of a laser as a clock. For this last topic I use some earlier work by myself [Phys. Rev. A 60, 4083 (1999)] and Berry and myself [Phys. Rev. A 65, 043803 (2002)] to show that a Heisenberg-limited laser with a mean photon number $\mu$ can synchronize $M$ independent clocks each with a mean-square error of $\sqrt{M} / 4 \mu$ radians ${ }^{2}$.
\end{abstract}

PACS numbers: 03.67.Mn, 42.50.Ar, 03.70.+k, 42.55.Ah 


\section{INTRODUCTION}

Recently, there has been an interesting controversy [1, 2, 3, 4, 5, 6, 7, 8, 9, in the quantum information and quantum optics community about the status of the first continuous variable quantum teleportation (CVQT) experiment [10]. It began when Rudolph and Sanders (RS) published a letter entitled 'Requirement of Optical Coherence for Continuous-Variable Quantum Teleportation' [1]. In it they argued that CVQT was not achieved in reference [10, and in fact that it cannot be achieved using a laser as a source of coherent radiation. They based their argument on their claim that a laser is not a source of coherent radiation, in the sense that the output of a laser is not a coherent state, but an equal mixture of coherent states with all possible phases.

It is true that any good quantum optics text (e.g. Refs. [11, 12]) will show that the stationary solution of the master equation for a laser yields a state which is an equal mixture of coherent states of all possible phases:

$$
\rho_{\mathrm{ss}}=\int \frac{d \phi}{2 \pi}|| \alpha\left|e^{i \phi \mid}\right\rangle\left\langle|\alpha| e^{i \phi \mid}\right|,
$$

where $|\alpha|^{2}=\mu$ is the mean photon number of the laser. As RS correctly point out, following Mølmer [13, this solution can also be interpreted as a mixture of number states, as

$$
\rho_{\mathrm{ss}}=\sum_{n} e^{-\mu} \frac{\mu^{n}}{n !}|n\rangle\langle n| .
$$

Thus, they argue, the description in reference [10] is invalid because it relies upon the 'partition ensemble fallacy' 14. That is, its analysis is carried using one partition of the ensemble (into coherent states) because it would not be valid in another partition (into number states).

An important motivation for RS is as follows [15. If one were to use the number state partition to describe the teleportation experiment, one would find that the entanglement necessary to do the teleportation is contained not in the conventionally recognized "entanglement resource" of twin-beam squeezing. Rather it would be a property only of the entangled resource plus an extra laser beam continuously shared between the two parties. Conventionally the laser is taken to be in a coherent state. If the phase of the coherent state is assumed to be unknown then this is equivalent to using the coherent-state partition. In this formulation the extra laser beam is regarded simply as establishing a time-reference. In the number-state partition, it appears as part of the entangled resource, which suggests that it is really another quantum channel, which is continuously open. Since quantum teleportation is supposed to take place in the absence of a quantum channel, this brings the validity of the experiment into doubt.

In this paper I will argue that the position of RS is unacceptable to any practising physicist or engineer, and that there are good reasons to say that sharing a laser beam is simply establishing a time-reference. That is, a laser is a clock, not a quantum channel. Van Enk and Fuchs (EF) 3, 4, have been the most active opponents to RS, but I will argue that their position is as flawed as that of RS, and for essentially the same reason. 
This paper is structured as follows. I will present my basic argument, that a laser is a clock, in Sec. 2. This is drawn largely from my presentation in reference [7]. The remaining sections (some of which are also based on this reference) are devoted to counter-arguments, and their flaws, as I see them. Some of these objections are published by others, some are unpublished (that is, personal communication), and some are hypothetical. I will use my responses to these objections to explore freely a number of interesting issues related to this question. In particular, I will discuss super-selection rules and their relation to conservation laws, quantum versus classical channels, and how good a laser is as a clock. The last point is quantified by the result that a quantumlimited laser with a mean photon number $\mu$ can synchronize $M$ independent clocks with a mean-square error of $\sqrt{M} / 4 \mu$ radians $^{2}$. This result, at least, I hope would have interested the honoree of this volume, Hermann Haus, as it relates precisely to the topic of his penultimate book [16].

\section{My Argument}

There is nothing wrong with the mathematics of RS. However, their letter suffers from a deep conceptual problem: they assume the existence of an absolute time or phase standard at optical frequencies. The reason they do so is that they do not wish to claim that CVQT at optical frequencies is impossible in principle. If they had claimed this then at least they would have established a consistent position, but also they would have also have displayed the practical absurdity of it. That is because if one insists on rejecting coherent optical sources, then one must discard much else besides.

To allow for the possibility of optical CVQT, RS must allow that the production of coherent states of light is possible in principle, by some means other than a laser. Indeed they say that 'We therefore assert that genuine CVQT requires coherent devices, that is, devices capable of generating true coherence, and these are not a feature of current [my emphasis] CVQT experiments.' If this were true then RS would have some basis for criticizing at least the formalism of reference [10]. It is the point of my argument here to show that even in principle there are no devices that can generate 'true coherence' any better than a laser.

Again emphasizing that their argument relies upon the possible existence of 'true coherence', RS say that they 'are of course not asserting that production of coherent states of light is impossible: basic quantum electrodynamics shows that a classical oscillating current can produce coherent states.' The first problem with this claim is that, as far as we know, the universe is quantal, not classical. There is no reason for believing in classical electrical currents. Nevertheless, a suitable quantum current would generate a coherent state of light to an arbitrarily good approximation, so I will not belabour this point.

A more serious problem is that it is not possible to produce even such a quantum current, in a sense that would pass the 'truth' test of RS. The natural oscillators at optical frequencies are the electrons in atoms. Without coherent light, we could still 
make the atom 'ring' by 'striking' it (with a free electron, for example). However, for this oscillation to have a definite phase the time of the collision would have to be known to an accuracy less than an optical cycle, of order $10^{-15}$ s. Otherwise one would have to average over all possible phases and one would be left with exactly the same problem as with the laser.

Achieving this accuracy would require a clock which ticks faster than $10^{15} \mathrm{~s}^{-1}$. Presumably this is the sort of 'technical challenge' that RS mention in the context of producing coherence by making a measurement on the gain medium of a laser. But to call it a 'technical challenge' is to miss the fact that it simply moves the problem back another step. How could we be sure that the clock has a definite phase? Perhaps it is fixed relative to another clock, but then we can ask the question again of that second clock. And so ad infinitum.

It is thus clear that the 'true coherence' as meant by RS is impossible to produce technologically. It cannot mean coherent relative to any clock, because their argument attacking the phase of a laser can be equally used to attack that of any clock. Therefore, if it means anything, 'true coherence' must mean coherent relative to an absolute time standard for the universe. Since such a hypothetical absolute time standard can never be measured, I would maintain that it is meaningless, and with it the idea of 'true coherence' in the sense of RS.

With no absolute time, all we can ever do is to use an agreed time standard, and measure phase relative to that. In this context, a laser beam is as good a 'clock' as any other. The electrodynamic coupling Hamiltonian $\propto \mathbf{p} \cdot \mathbf{A}(\mathbf{x})$ allows, in principle, the laser clock to influence the motion of a charged particle with position $\mathbf{x}$ and momentum p. In physical terms, when the electric field points up, an electron is deflected down, and vice versa. This can be used to synchronize the laser with any material clock.

Once material particles are synchronized with the laser field, they could then be synchronized with any other clock based on any gauge boson field, via analogous coupling Hamiltonians [17]. This includes gravitational and nuclear force fields, but not any of the "second quantized" fields of composite bosons such as mesons or Hydrogen atoms. Material oscillations of course must involve a force field, and hence also involve the oscillation of a gauge boson field of some sort. The fungibility of the time standards based on different gauge boson fields makes all of these time-keepers equivalent, and justifies calling them 'clocks'. This is not an empty definition, as the other (non-gauge) boson fields, are not fungible time keepers. For example, an atom laser beam cannot be a clock in this sense. Its phase can only be defined or measured relative to another atom laser beam of the same species [18.

Fundamentally, the difference between (for example) an atom laser and an optical laser is that the atom laser field arises from the quantization of identical particles, not the quantization of a field. This explains the difference in the way the field appears in a Hamiltonian. In coupling Hamiltonians a gauge field can appear linearly. By contrast, the field describing particles always appears bilinearly, and in conjugate pairs; if one particle is destroyed, another is always created. If one is familiar with Feynman 
diagrams, then the difference can be understood from the fact that every vertex involving a particle has two lines for particles, but one (wiggly) line for the gauge field [17. In the standard model, the particles (in this sense of the word) are fermions, but the consequences is seen in the non-fungibility of the phase of the bosonic field for composite particles such as in an atom laser.

The above arguments lead inevitably to the conclusion that in quantum optical experiments there is no necessity to consider, even hypothetically, any time-keeper beyond the laser which serves as a phase reference. No other clock is superior in any fundamental sense. Now by definition a laser beam is perfectly coherent relative to itself (ignoring experimentally negligible phase and amplitude fluctuations, and transverse mode incoherence). Thus, the phase reference laser beam in the teleportation experiment is in a coherent state. It is not a coherent state of unknown phase, it is the coherent state $|\sqrt{\mu}\rangle$, with zero phase (relative to itself).

To reiterate, I am not advocating an ignorance interpretation of the ensemble in equation (1). It would be illegitimate to do so. There is no homunculus preparing the laser in a coherent state of some particular phase, but refusing to divulge that knowledge to us. It is we who set the phase of the laser to be zero by calling it a clock. There is no process that will make a coherent state in any stronger sense, and no need for for any stronger sense. There is no unmet need for optical coherence in continuous-variable quantum teleportation.

\section{Objection: We experience absolute time}

The first obvious objection that might be voiced is that as conscious beings we experience the flow of absolute time directly, and so give it meaning. Admitting the validity of this temporal experience (which does not go without saying [19]), this argument nevertheless cannot work to establish optical coherence. We cannot simply look at a clock ticking every $10^{-15} \mathrm{~s}$ and verify that it has a definite phase, because we cannot perceive anything in $10^{-15} \mathrm{~s}$.

My rebuttal applies not only to optical frequencies. Experiments show that our perception of time has a resolution in the range of tens or even hundreds of milliseconds [20]. Thus we cannot establish the absolute phase of any oscillator of frequency greater than a few tens of Hertz. At higher frequencies we can only establish the phase of one oscillator relative to another oscillator. This conclusion is not altered by oscillations obtained by frequency combs or $2^{n}$-upling [21]. The timing of the zeros of the highest harmonic can be no more accurately defined than that of the fundamental.

From personal observation, discussions between physicists about the existence of 'true coherence' or 'mean fields' often end with one party waving an arm up and down, intimating that by so moving an electric charge, a mean field would be produced. But that appeal fails precisely when the frequency is too fast for us consciously to move any part of our body at that frequency. That is, it again fails for frequencies greater than a few tens of Hertz. 
There are many qualitative differences between the way radiation is generated, or detected, across the frequency spectrum. But the only location for a fundamental (if fuzzy) dividing line in frequency between absolute and relative phase, or between 'true coherence' [1] and 'convenient fictions' 13. would be between oscillations we can observe directly and those we cannot. If this division is unpalatable that is because it relies upon the notion of absolute time. By abandoning this ill-conceived notion, the dividing line between supposed 'truth' and supposed 'fiction' disappears. There is only phase relative to clocks, be they human, mechanical, radio, optical and (in the future) so on.

\section{Objection: A laser is not "really" in a coherent state}

One would be entitled to be puzzled by the fact that I began by seeming to accept that a laser at steady state is described by a mixture, as in equations (1) or (2), but ended by claiming that it is in a pure state $|\sqrt{\mu}\rangle$. This certainly troubled Fuchs [22]. I will give my resolution below, but before that I should say a few words about the published opinions of Fuchs, with van Enk, [3, 4] on RS [1]. My understanding of their papers is that they are an explicit calculation (which can be done analytically because they ignore the laser gain process) showing how a laser beam, without an absolute phase, can function as a phase reference. Specifically, they show how the phase information can be distributed and how there is no harm in regarding the phase as real. This is essentially the same point originally made by Mølmer, that the laser phase is a 'convenient fiction' [13]. The analysis of EF gives a rigorous information-theoretic definition of 'convenience', in terms of the quantum de Finetti theorem [4]. However, it could well be questioned whether convenience, however rigorously defined, is sufficient for a dispensation from the ban on using the 'partition ensemble fallacy'.

The real lesson that should have been drawn from the analysis of $\mathrm{RS}$ is that quantum teleportation can be, and should have been, defined operationally, so that the reality of the laser phase would have been irrelevant. The reality of the laser phase can nevertheless be defended, as I have done, on the grounds that it is no less real than any other phase. However, this is not the position of EF. Instead, they base their entire analysis on an unquestioning acceptance of equations (1) and (2) [23]. I call this acceptance unquestioning because they follow RS in assuming that there is such a concept as 'true coherence', and that this is something that a laser lacks by virtue of being in a mixture of the form of equations (1) and (2).

Indeed, in reference [3], EF directly imply that there is a time standard more real than that offered by the laser itself:

However, recent developments 21] may make it possible to compare the phase of an optical light beam directly to the phase of a microwave field. Using this technique, the only further measurement required ... is a measurement of the absolute phase [my emphasis] of the microwave field, which is possible electronically. This measurement would create an optical coherent state from a standard laser source for the first time. 
The implication is that an electronic measurement somehow makes the phase real, which it was not when it was an optical phase. As I have argued above, there is no reason to regard the laser phase as any less real than any other phase. There is nothing gained in, and no need for, appealing to any other clock in order to say that the laser is in a coherent state.

Thus I can finally state the resolution of the puzzle with which I began this section. In general, any state of an oscillator is meaningless without a specification of the time reference (i.e. clock) by which it is defined. (The exception is an energy eigenstate.) A state may have an ill-defined phase relative to one clock, but be perfectly coherent relative to another one. In particular, if the laser itself is the clock then by definition it is coherent with respect to itself so that it should be described by a pure state $|\sqrt{\mu}\rangle$ of zero phase. It is ironic that one (Fuchs) whose mantra is that quantum states are states of knowledge (see for example reference [24]) should neglect the epistemic component to a state like that in equation (1).

In fairness to Fuchs' position, I should reproduce a further argument he has communicated to me [22]. It is based on a theorem discovered by Schrödinger [25] and called by him "steering" (see also reference [26]). This theorem states that if a system is in a mixed state by virtue of being entangled (that is, there is a pure state in the larger Hilbert space of the system and its environment) then there is a way to measure the environment such as to "steer" the system into any one of the ensembles of pure states that represents that mixed state. Since the average state of the system is unchanged regardless of which ensemble is used to represent it, it is arguable as to whether steering is an appropriate term. Nevertheless its application in this case is clear. If the laser used by Alice and Bob for CVQT is in a mixed state purely by virtue of being entangled with its environment, then there would be a way to for a third independent observer (call him Chris) to measure that environment so as to collapse the laser into a number state $|n\rangle$, with $n$ randomly chosen from the probability distribution in equation (2). If Chris knows the laser to be in state $|n\rangle$, then how can Alice and Bob claim that it, the clock, is in a coherent state $|\sqrt{\mu}\rangle$ ?

One may well imagine the response of Alice and Bob to Chris' attempt to carry out this measurement: "get your filthy hands off our clock!" By measuring the environment so as to collapse the laser into a number state, Chris would prevent it from being used for its desired purpose, namely to be a clock. Chris would have to detect all of the light emitted by the laser, so there is no way Alice and Bob could use it to establish a phase reference. Even if Chris did a quantum-non-demolition measurement of the photon number of the light, this would destroy its phase and so still prevent it from being used to establish a definite phase between Alice and Bob. It is not surprising that, when it cannot be used as a clock, it is not valid to claim that a laser has a well-defined phase. But when a laser is being used as the clock for the experiment there is no way Chris can know which number state it is in, and no reason for Alice and Bob not to describe it by a coherent state $|\sqrt{\mu}\rangle$.

To sum up my position: a quantum state is not only a state of knowledge, it is a state 
of purpose. That is, it is not only what I know about a system, but also how I intend to use it, that determines how it is described formally. To ignore this fact is to take the mathematical formalism of quantum mechanics too seriously. Operationally, sharing a laser is simply clock synchronization. There is no puzzle here, and our formalism should reflect that. To argue otherwise may be interesting philosophy, but it is not interesting physics.

Note finally that allowing a quantum state to be a state of purpose is not tantamount to turning the partition ensemble fallacy into the partition ensemble truth. Of course any experimentalist can invent a purpose that allows them to claim that a given mixed state is "really" in a pure state drawn randomly from one particular ensemble. But if the experiment genuinely serves a purpose, and the outcomes depend upon the state in the experimenter's mind, then the randomness in the draw will adversely affect the experiment regardless of the experimenter's mind-set. It is precisely because no experiment is affected by the supposed randomness in the phase of the laser (if it is being used as a time reference) that makes it possible to describe the laser by a single state $|\mu\rangle$, and not an ensemble. A similar point is made by Nemoto and Braunstein [6].

\section{Objection: Conservation of energy implies a superselection rule for photon number}

A super-selection rule (SSR) for a particular observable $\hat{O}$ is a restriction on the possible operations that can be implemented physically. Roughly, it says that it is impossible to produce states that are superpositions of eigenstates of $\hat{O}$ with different eigenvalues. For a more precise exposition see reference [27.

Neither RS, nor EF, nor I, claim that there is a SSR for photon number. For RS, and $\mathrm{EF}$, this is because they believe that there is a way, with a classical field (RS), or a microwave field (EF), to produce such optical coherences. For my part, I claim that whether or not a phase is a superposition or a mixture depends upon how it is used. If it is used as a clock, then it is coherent by definition. However, the notion that conservation of energy implies a SSR for photon number does exist in the community. If it were true then this would present a problem for my argument, and in fact would be a physical basis for preferring the ensemble in equation (1) to that in equation (2). Therefore in this section I will rebut that notion.

To begin it is necessary to clear up some misconceptions. I will start with a bold claim: SSRs should be applied only to non-conserved quantities. That is not to say that SSRs are unrelated to conserved quantities, but the relation is more subtle, and I have not seen a decent exposition elsewhere. Here is mine. It is obvious that one cannot create superpositions of different values of conserved quantities. Conserved quantities do not change, by definition. If initially the system were in an eigenstate of a quantity then it would of course be impossible to create a superposition, because this would be equivalent to changing $\hat{O}$. The same is true for initial mixtures. Thus there is no need 
to invoke an SSR to say that creating superpositions is impossible. The conservation law already tells one this. The only useful SSR is one that applies to non-conserved quantities.

The relation between SSRs and conservation laws is as follows. If and only if a conserved quantity $\hat{C}$ can be written as

$$
\hat{C}=\hat{c}_{1} \otimes \hat{1}_{2} \otimes \hat{1}_{3} \cdots \otimes \hat{1}_{N}+\hat{1}_{1} \otimes \hat{c}_{2} \otimes \hat{1}_{3} \cdots \otimes \hat{1}_{N}+\cdots+\hat{1}_{1} \otimes \hat{1}_{2} \otimes \cdots \hat{1}_{N-1} \otimes \hat{c}_{N}
$$

then it is possible to derive a SSR (in fact, $N$ of them). Namely, these are that it is impossible to produce superpositions of states having different eigenvalues of $\hat{c}_{k}$, for $k=1, \cdots, N$. This follows from the presumption that the initial state (of the universe, or of the laboratory if one wishes to be more modest) is an eigenstate, of $\hat{C}$. Note that $\hat{c}_{k}$ is not in general conserved. Nevertheless it obeys a SSR, because if a state were a superposition of different eigenstates of $\hat{c}_{1}$, for example, then by the structure of equation (3) it would be a superposition of different eigenstates of $\hat{C}$, and that is forbidden by the initial conditions and the conservation of $\hat{C}$.

An example may help. Total momentum $\hat{P}$ is a conserved quantity. Furthermore, total momentum is composed of a sum of momenta of individual particles and field modes, as in equation (3). These individual momenta $\hat{p}_{k}$ are not conserved, as the particles and fields interact. But, if the universe were to have begun in a momentum eigenstate, then there would be a SSR for every individual momentum. That is, no particle could be localized, because it would always be a mixture of momentum eigenstates, never a superposition. This is simply because the initial state of the universe would be completely delocalized also.

Perhaps this is a poor example, because saying that the universe began in an eigenstate of momentum is arguably a meaningless statement. This is because it is meaningless to discuss a spatial reference frame in the absence of the matter of the universe, just as it is meaningless to discuss the phase of an oscillator in the absence of a clock. Thus, one could argue that, relative to itself, the universe is not in a momentum eigenstate, since it is clearly somewhere definite, namely exactly on top of itself. The same argument would hold for spatial orientation, and the corresponding SSR for angular momentum. By contrast, claiming that the universe is in an eigenstate of total charge is perhaps meaningful, at least in some cosmologies. Since total charge is also the sum of charges of subsystems, a SSR for the charge of such systems (e.g. a certain volume of space) is arguably better justified.

Regardless of whether this derivation of SSRs from conservation laws and initial conditions is ever justified, it is certainly not justified for energy. The total energy $\hat{H}$ of the universe may be conserved, but it is not of the form of equation (3). If it were of this form then that would mean that the universe would consist of some disjoint parts which have no interaction whatsoever with one another. So really, the Hamiltonian $\hat{H}$ would be describing a number of universes, not one universe. Thus, there is no energy SSR, so a photon number SSR cannot be derived from it.

In the quantum optical case, it is the interaction Hamiltonian between the 
electromagnetic field and charged particles that prevent the Hamiltonian being of the form of equation (3). This is not an inconvenience that might be ignored. Rather, is crucial to the fact that an electromagnetic field can be a clock. As noted in Sec. II, it is the $\mathbf{p} \cdot \mathbf{A}(\mathbf{x})$ coupling that makes the electromagnetic phase reference fungible with all other clocks.

\section{Objection: A laser beam needs a quantum channel}

Even if one accepts all of my above arguments, one may still wonder about the initial issue which at least partly motivated RS, namely that the laser beam is acting as a quantum channel. Operationally this problem could be easily removed by defining quantum teleportation to require that the phase reference channel, be it quantum or classical, must not be open during the teleportation itself. That is, setting up the time reference would, like the sharing of entanglement, be part of the setup prior to teleportation. This would certainly be possible in principle although in practice it would be inconvenient as it would require maintaining the optical phase of a light field (such as that in a high-finesse cavity) for a relatively long time (perhaps many $\mu$ s) on each side.

However, it is interesting nevertheless to ask the question, is a quantum channel necessary to share a phase reference? To answer this one needs a way to distinguish a classical and a quantum channel. The obvious definition is that used, perhaps for the first time, in reference [28] : a classical channel is a channel that does not permit the sending of quantum information. Specifically, a classical channel completely decoheres the system in some basis. That is, it diagonalizes the state matrix in that basis, suppressing the coherences. This is precisely what was done in the NMR quantum teleportation of reference [29].

Since the coherent states are non-orthogonal and overcomplete:

$$
\int d^{2} \alpha|\alpha\rangle\langle\alpha|=\pi \hat{1}
$$

it might be thought that it would be impossible to send coherence down a classical channel. It is true that it is impossible to send a coherent state down a classical channel and have it emerge unscathed. However, to send coherence (i.e. a phase reference) this is not necessary. All that is necessary is for the emerging state to have a large coherent amplitude which is approximately equal to that of the coherent state that entered. This is quite possible.

Consider as a crude example the states $\left\{\left|q_{n}, p_{m}\right\rangle: n, m\right.$ integers $\}$ defined in the $\hat{q}$-representation as

$$
\left\langle q \mid q_{n}, p_{m}\right\rangle=\Delta^{-1 / 2} \chi_{\left[q_{n}-\Delta / 2, q_{n}+\Delta / 2\right]}(q) \exp \left(-i q p_{m}\right) .
$$

Here $q_{n}=\Delta n$ and $p_{m}=2 \pi m / \Delta, \chi_{S}(x)$ is the characteristic function, equal to 1 when

$x \in S$ and 0 otherwise, and $\hat{q}=\left(\hat{a}+\hat{a}^{\dagger}\right) / \sqrt{2}$. These states have a mean coherent amplitude:

$$
\left\langle q_{n}, p_{m}|\hat{a}| q_{n}, p_{n}\right\rangle=\left(q_{n}+i p_{m}\right) / \sqrt{2}
$$


The variance (and indeed all moments) are finite for $\hat{q}$. For $\hat{p}=-i\left(\hat{a}-\hat{a}^{\dagger}\right) / \sqrt{2}$ the variance (and indeed all moments) are infinite. Nevertheless the probability distribution $P(p)$ is peaked (with a width of order $\Delta^{-1}$ ) about $p=p_{m}$. Thus with a choice of $\Delta \sim 1$ this is a state with well-defined coherent amplitude, and a fair overlap with a coherent state $|\alpha\rangle$ with $\alpha$ close to $\left(q_{n}+i p_{m}\right) / \sqrt{2}$.

The states $\left\{\left|q_{n}, p_{m}\right\rangle: n, m\right\}$ form an orthonormal set with the completeness relation

$$
\sum_{n, m}\left|q_{n}, p_{m}\right\rangle\left\langle q_{n}, p_{m}\right|=\hat{1}
$$

Thus they can be used to define a classical channel, by specifying the trace-preserving operation on the channel

$$
\rho \rightarrow \mathcal{O} \rho=\sum_{n, m=-\infty}^{\infty}\left|q_{n}, p_{m}\right\rangle\left\langle q_{n}, p_{m}|\rho| q_{n}, p_{m}\right\rangle\left\langle q_{n}, p_{m}\right|
$$

Clearly a pure coherent state $|\alpha\rangle$ will become mixed by this channel. However, as long as it is a good phase reference, so that $|\alpha| \gg 1$, the state that emerges

$$
\sum_{n, m}\left|\left\langle q_{n}, p_{m} \mid \alpha\right\rangle\right|^{2}\left|q_{n}, p_{m}\right\rangle\left\langle q_{n}, p_{m}\right|
$$

will be dominated by states with $\left(q_{n}+i p_{m}\right) / \sqrt{2} \simeq \alpha$. Thus the state still carries the phase information necessary to establish a phase reference.

It might be objected that in the experiment there is no channel which is described by the operation (8), and thus that in the experiment there is a quantum channel, not a classical channel. But this seems unfair. Say an experimenter E claims to be using a channel as a classical, not a quantum, channel. Say a skeptic S challenges the validity of an experiment by claiming that actually it is being used as a clandestine quantum channel. E comes back and says, "Okay, to prove it is being used as a classical channel, I will repeat the experiment while allowing you $(\mathrm{S})$ to decohere the channel in this basis which I specify." S says "But I don't know how to perform that operation." Says E, "Tough — in a duel the challengee chooses the weapons. The onus is on you if you want to invalidate my experiment."

Following on from this argument, the stymied challenger will (as I well know) continue to raise the objection that without the decohering mechanism, an optical channel (e.g. free space or a fiber) can be used to send quantum information. In a genuinely classical way to synchronize clocks, this would not be the case. For example, one could send marbles down a pipe so that they arrive at the far end at regular times, separated by $10^{-15}$ s.

The error with this argument is that it assumes that, just because we ordinarily describe marbles as classical objects, that they really are classical objects. If one allows for the existence of classical objects by fiat, then by fiat I would claim that a laser beam is a classical object. Certainly to an ordinary laser physicist, as opposed to a quantum optician, laser beams are classical objects, and the former would laugh at the idea that synchronizing clocks using laser beams was sending quantum information. 
Allowing instead that marbles are quantum objects, how is a free space channel used for clock-synchronization by marble arrival-time a quantum channel? The answer is in the longitudinal momentum modes of the marbles. If the marbles arrive at definite times (as was the original assumption) then they must have well-defined wave-packets in position space. That is, they must be superpositions of different momenta. In principle, this could be used to encode quantum information, as the superposition of momentum eigenstates is preserved by free evolution.

Just as in the laser case, it would be possible to defend the marble channel as a classical channel, by choosing some basis set of orthogonal states along which to decohere the channel. These could not be momentum eigenstates, as this would make the position of the marbles completely delocalized, so all timing information would be lost. Nor could it be position eigenstates, as these are states of infinite energy which would immediately become delocalized also. Rather, just as in the laser case, what would be required is states localized in phase space with some suitably chosen position width $\Delta$.

No one would take you seriously if you insisted that an experiment using marble-timing to synchronize clocks is really using quantum information, unless the experimenters make sure they decohere the longitudinal quantum state of their marbles completely along some basis. The point is that there is absolutely no difference using a laser. Exactly the same issues will always arise, if one thinks carefully, regarding the preparation and dissemination of states which can be used to synchronize clocks.

\section{Objection: A laser cannot synchronize arbitrarily many parties}

The final objection I will consider is that a laser beam is not a clock because it cannot establish a time standard between arbitrarily many parties. Eventually it will run out, or, if it is a continuous beam, its finite linewidth will mean that the later part of the beam has a random phase relative to the former part. This is of course true, and the fundamental limits are set by the finiteness of the excitation of the laser mode and laser

gain medium [30]. This excitation may be very large (measured in units of $\hbar \omega$ ), but is not infinite.

Once again, the mistake in this reasoning is to ignore the quantum nature of other clocks, simply because we are used to describing them classically. All physical clocks have finite excitation, and cannot be used to synchronize arbitrarily many parties. Low frequency material clocks typically have huge excitations (in units of $\hbar \omega$ ), so we are not used to worrying about their finiteness. A similar point has been made in reference [31].

At optical frequencies, a laser is actually the best clock we have. In fact, at the National Institute of Standards in Boulder, Colorado, the next generation of an "atomic clock" in development is actually an optical clock using a laser [32. As pointed out in reference [8], this is a powerful argument in support of my view that a laser already produces a coherent state. Once a laser becomes the basis for the world's time standard, then establishing the phase of a laser relative to a mechanical oscillator will fix the phase of the latter, rather than the former. 
Nevertheless, it is an interesting question to ask precisely how good could a laser be as a time standard? That is, what are the quantum limits to how many clocks could be simultaneously synchronized with the standard laser? Clearly for an infinitely large laser the answer is that there is no limit. But for a laser with a finite excitation, there must be a definite answer, and to my knowledge, it has not been given before. The answer is that for a laser with a mean excitation number $\mu$, the number $M$ of clocks that can be synchronized with a mean-square error $(\delta \phi)^{2}$ (in radian $^{2}$ ) satisfies

$$
(\delta \phi)^{2} \geq \sqrt{M} / 4 \mu
$$

This expression, to me at least, is not an obvious one. Readers may wish to see if they can derive it (ignoring factors of order unity) from an argument which is simple (in comparison with the derivation presented below), and to inform me if they can.

The first point to note is that this expression cannot be derived simply by considering the dividing of a coherent state of amplitude $\sqrt{\mu}$. In the large-amplitude limit, the phase variance of a coherent state $|\alpha\rangle$ is equal to $1 / 4|\alpha|^{2}$ (see e.g. reference [12]). Thus if the coherent state $|\sqrt{\mu}\rangle$ were split coherently into $M$ coherent states of amplitude $\sqrt{\mu / M}$, the phase variance of each one individually would be

$$
(\delta \phi)_{\text {split }}^{2} \sim M / 4 \mu
$$

Note the difference from the above by a factor of $\sqrt{M}$. For $M$ large, which is the case we are interested in, this phase variance may be thought of as the mean square phase error relative to the original coherent state $|\sqrt{\mu}\rangle$ which has negligible phase uncertainty itself. However these small coherent states may be amplified, the phase error relative to the original cannot be reduced.

A second point to note is that equation (10) can also not be derived by considering the optimal cloning of a single coherent state 33. Such cloning (which can be achieved by linear amplification followed by splitting) results in $M$ copies with a phase variance equal to

$$
(\delta \phi)_{\text {clone }}^{2} \simeq \frac{3-2 / M}{4 \mu} \sim \frac{3}{4 \mu} .
$$

This is independent of $M$ for large $M$, quite unlike equation (11) or equation (10). The fact that this limit is far smaller than equation (10) may seem to undermine my claim that equation (10) is the quantum limit for phase locking a laser. The point is that equation (10) applies to the situation of a given laser of fixed mean number. To produce an arbitrarily large number of copies by optimal cloning requires an arbitrarily powerful linear amplifier. Obviously that amplifier could be used to replace (or enhance) the original laser, producing a new laser with an arbitrarily larger mean photon number. It is therefore not surprising that cloning breaks the limit of equation (10).

Another difference between the two cases (synchronizing to a laser, and distributing a coherent state) is that the laser maintains its mean photon number even as it generates an output that can be shared amongst many parties. The price paid for this is that the phase of the laser does not remain constant over time. Rather, it diffuses. The object of phase locking is to lock to the current phase, rather than to the initial phase. 
To derive equation (10) it is necessary to answer two questions. First, what is the quantum limit to the rate of laser phase diffusion? Second, what is the quantum limit to how well a second clock can be synchronized to a continuous signal with a diffusing phase? That is the content of the next two subsections.

\subsection{Quantum limits to laser phase diffusion.}

This topic will in most readers' minds conjure up the names of Schawlow and Townes who introduced the idea of an "optical maser" some 45 years ago [34. Actually, the expression in their paper for the quantum-limited laser linewidth is irrelevant for most modern lasers. That is because it was derived in the days before good optical cavities, and hence implicitly assumes that the atomic linewidth $\gamma$ is much smaller than the (FWHM) cavity linewidth $\kappa$. This is discussed in detail in reference 30.

In more recent times, the term "Schawlow-Townes limit" has commonly been used to refer to the quantum limit for the linewidth of a laser in the limit that the atomic medium can be adiabatically eliminated (which requires $\kappa \ll \gamma$ ). I have argued 30] that this use is unwise (although I was guilty of it myself [18]). Regardless of what it is called, the quantum-limited linewidth $\ell$, far above threshold, is given by

$$
\ell_{\mathrm{SQL}}=\frac{\kappa}{2 \mu} \text {. }
$$

Here $\mu$ is, as above, the mean photon number of the laser. I will call this the standard quantum limit (SQL) linewidth. As I will discuss below, it is not the ultimate limit to the linewidth set by quantum mechanics, which I will call, following standard practice in other areas, the Heisenberg limit (HL) to the linewidth.

Most older textbooks [35, 11, 36] quote the result in equation (13), or one which reduces to it in the appropriate limit of neither reabsorption nor thermal photons. All three of these books attempt to explain this linewidth in terms of the noise added by the spontaneous contribution to the (mostly stimulated) gain of photons from the atomic medium. Far above threshold the amplitude of the laser is essentially fixed. Thus the effect of spontaneous emission noise is supposedly to randomly perturb the laser phase (relative to some hypothetical time standard). This can be modeled by the equation

$$
\dot{\varphi}=\sqrt{\ell} \xi(t),
$$

where $\xi(t)$ is Gaussian white noise satisfying $\left\langle\xi(t) \xi\left(t^{\prime}\right)\right\rangle=\delta\left(t-t^{\prime}\right)$. It is easy to show 35. that this gives rise to a Lorentzian lineshape of linewidth $\ell$.

It turns out [30] that these explanations are strictly incorrect. Attributing the phase diffusion solely to the gain mechanism is an artifact of thinking in terms of normallyordered operator products. That is, it results from using (implicitly in most cases) the Glauber-Sudarshan $P$ function [12] as a true representation of the the fluctuations in the laser mode field. The $P$ function is of course no more fundamental than the $Q$ function 12, which is a representation based on anti-normally ordered statistics. If one were to use the $Q$ function as an aid to intuition, one would find that it is the loss process that is 
wholly responsible for the phase noise. Of course the rate of phase diffusion would agree with that from the $P$ function, at least in steady-state where loss and gain balance.

If one asks a question about phase diffusion, the only objective answer will come from using the phase basis itself. This is far more difficult than using the more familiar phase-space representations, but some approximate results have been obtained [37]. These show that, at steady state, the phase diffusion has equal contributions from the loss and gain process. The same result occurs from a Wigner function calculation 37. This is not surprising since symmetrically ordered moments are known to closely approximate the true moments for the phase operator for states with well-defined amplitude 38. A linearized treatment using Heisenberg equations also says that equal contributions come from loss and gain. For a transparent exposition, see Yamamoto and Haus 39], Sec. VI.

The fact that standard phase diffusion comes equally from the loss and gain processes suggests that the SQL to the laser linewidth, $\ell_{\mathrm{SQL}}$ of equation (13), may not be the ultimate quantum limit. The contribution from the loss mechanism is unavoidable. A laser, at least in useful definitions [18, requires linear damping of the laser mode (here with decay rate $\kappa$ ) in order to form an output beam. However, it may be that the standard gain mechanism could be replaced by some other gain mechanism that still yields the same steady state (1), but which causes less phase diffusion. That is, we assume that the state matrix $\rho$ for the laser mode obeys the master equation

$$
\dot{\rho}=\mathcal{L}_{\text {gain }}\left[\hat{a}, \hat{a}^{\dagger}\right] \rho+\kappa\left(\hat{a} \rho \hat{a}^{\dagger}-\frac{1}{2}\left\{\hat{a}^{\dagger} \hat{a}, \rho\right\}\right)-i \omega\left[\hat{a}^{\dagger} \hat{a}, \rho\right],
$$

where the gain term is time-independent (that is, involves no external driving), but may be different from the standard laser gain term. The HL to the laser linewidth from such an equation could be as small as one half of the standard limit.

It turns out that it is indeed possible to find a gain Liouvillian $\mathcal{L}_{\text {gain }}\left[\hat{a}, \hat{a}^{\dagger}\right]$ which, contrary to the standard term, adds no phase noise [30]. For interest, it is given explicitly by

$$
\mathcal{L}_{\text {gain }}\left[\hat{a}, \hat{a}^{\dagger}\right] \rho=\kappa \mu\left[\hat{a}^{\dagger}\left(\hat{a} \hat{a}^{\dagger}\right)^{-1 / 2} \rho\left(\hat{a} \hat{a}^{\dagger}\right)^{-1 / 2} \hat{a}-\rho\right] .
$$

Moreover, there is physical mechanism by which this gain could be realized, using adiabatic transitions in cavity QED [30. The HL laser linewidth is thus half the SQL:

$$
\ell_{\mathrm{HL}}=\frac{\kappa}{4 \mu} .
$$

It is actually quite easy to derive this limit from an elementary argument [30, which I will reproduce here because of its brevity. Using the quadrature operators $\hat{q}$ and $\hat{p}$ as earlier, one has for coherent states $V(q)=V(p)=1 / 2$, where $V$ denotes the variance. From equation (1), the laser mode can be assumed to be in such a state at some time 0 , with mean amplitude $\sqrt{\mu}$. Let the mean amplitude of the coherent state be real and positive so that $\bar{q}=\sqrt{2 \mu}$ and $\bar{p}=0$. For $\mu \gg 1$ the fluctuations in $\hat{q}$ can be ignored compared to its mean, so that the phase variance is thus

$$
V(\varphi) \simeq V\left(\operatorname{artan} \frac{p}{\bar{q}}\right) \simeq \frac{V(p)}{\bar{q}^{2}}=\frac{1}{4 \mu} .
$$


Now the effect of linear damping as in equation (15) for an infinitesimal time $d t$ is to reduce the mean photon number of the coherent state from $\mu$ to $\mu(1-\kappa d t)$. Thus the change in the phase variance is

$$
d V(\varphi)=\frac{\kappa d t}{4 \mu}
$$

A noiseless gain process will return the mean photon number to $\mu$ without increasing the phase noise. Therefore the phase variance increases at least as fast as $\kappa / 4 \mu$, which is precisely what results from equation (14) with $\ell=\kappa / 4 \mu$.

\subsection{Quantum limits to phase locking}

From the above, we know that we can model the output of a laser as a coherent state with a phase stochastically evolving according to equation (14). The power of the laser output beam is $P=\hbar \omega \kappa \mu$. It is more convenient to measure power in photons per second, for which I will use the symbol $f$. If the laser output is split equally between $M$ parties, for the purposes of clock synchronization, then the power in each beam is $f=\kappa \mu / M$.

The question is then, what is the best way to synchronize one's own clock (e.g. a laser) to a continuous beam of power $f$ with a phase diffusing according to equation (14). I will assume that each party has their own laser, with an arbitrarily large photon number, so that the rate of phase diffusion of these lasers is negligible compared to the rate of phase diffusion $\ell$ of the source laser (to which all others are synchronized). In practice, one would expect the reverse situation; that is, one would want the standard laser to have the lowest noise. However for the purposes of determining the limit to how good a given, finite, laser can be as a phase reference, it is necessary to consider the above situation.

One possibility for clock synchronization, considered in reference [8], is to use the beam as a seed to one's own laser. As Heydari and Björk show, this is certainly adequate, but they do not consider its quantum limits. Since the gain term in a standard laser does introduce unnecessary phase noise (as discussed above) it seems unlikely that this process would be the optimal one. It is possible that a laser with a non-standard gain term, as in equation (16), would be optimal, but the analysis of that question is beyond the scope of this paper.

Rather than using the source beam to seed the local laser, the phase of the latter can be locked to the former by measuring the phase difference between them, and adjusting the phase of the latter so as to eliminate as far as possible this phase difference. This is precisely the problem considered in reference [40]: to estimate continuously the phase of a CW beam with a finite power and non-zero linewidth (i.e. finite phase diffusion rate).

It turns out, unsurprisingly [41, that the optimal technique for estimating the phase using conventional experimental techniques (linear optics and photodetection) is an adaptive one. That is, it is necessary to use past measurement results (photocurrents) 
to control the present conditions of the measurement in order to obtain the maximum information about the phase. Attaining the Heisenberg limit of phase locking requires quite precise control of the feedback gain the in the locking loop. This has recently been demonstrated experimentally for the case of single-shot (as opposed to continuous) phase estimation [42, using the algorithm in reference 43 .

For coherent light (i.e. non-squeezed light) as we are considering here, using a nonadaptive technique, the MSE in the best estimate for the phase $\phi$ is, in steady state, 40

$$
(\delta \phi)_{\mathrm{SQL}}^{2}=\frac{1}{\sqrt{2 N}}
$$

Here $N=f / \ell$ is a dimensionless parameter which (up to factors of order unity) is equal to the number of photons in the beam per coherence time, or, equivalently, the peak photon current per unit bandwidth. We have assumed $N \gg 1$ because this is necessary to obtain good clock synchronization. Note that the phase variance scales as $N^{-1 / 2}$, not $N^{-1}$ as might be naively expected.

An example of non-adaptive measurement technique is ambi-quadrature homodyne detection, where the beam is split in two, with one half being used to perform a measurement of one quadrature and the other half used to perform a measurement of the orthogonal quadrature. This non-adaptive technique is wasteful because it obtains information about the amplitude equally as well as about the phase, of the beam. Because these are complementary quantities, obtaining the amplitude information halves the amount of phase information that can be obtained. This is the best that can be done non-adaptively.

An adaptive technique ensures that ones obtains, at least approximately, only phase information. As in the discussion in the preceding subsection on laser gain mechanisms, the boon granted by eliminating unnecessary phase uncertainty is modest. Specifically, the adaptive technique yields [40]

$$
(\delta \phi)_{\text {adapt. }}^{2}=\frac{1}{2 \sqrt{N}}
$$

Again, this result can be derived from a relatively simple argument [41], which I will reproduce since it has not appeared in a refereed journal before.

Denote the amplitude of the coherent beam by $\alpha=\sqrt{f}$, and its phase by $\varphi$. To make a phase sensitive measurement it is necessary to interfere this beam with a local oscillator (from the laser to be locked). Assuming the local oscillator to be much stronger than the signal beam, the resulting photocurrent, suitably scaled, is [43]

$$
I(t)=2 \alpha \cos [\Phi(t)-\varphi(t)]+\zeta(t),
$$

where $\Phi(t)$ is the local oscillator phase, and $\zeta(t)$ is another Gaussian white noise term, independent of the $\xi(t)$ in equation (14), with unit spectral power. It can be thought of as local oscillator shot noise, or vacuum noise.

The optimal non-adaptive estimation can be achieved from such a measurement by having $\Phi(t)$ vary rapidly over all phases. For example, in heterodyne detection 
with a detuning $\Delta \gg \sqrt{\alpha \ell}, \Phi$ varies as $\Phi(t)=\Phi(0)+\Delta \times t$. From equation (22), we would expect better sensitivity if one were to choose $\Phi(t)$ to maximise the slope of $\cos [\Phi(t)-\varphi(t)]$. That is, one should set $\Phi(t)=\check{\varphi}(t)+\pi / 2$, where $\check{\varphi}(t)$ is one's best estimate of the phase $\varphi(t)$. This ensures that that

$$
\begin{aligned}
I(t) & =2 \alpha \sin [\varphi(t)-\check{\varphi}(t)]+\zeta(t) \\
& \simeq 2 \alpha[\varphi(t)-\check{\varphi}(t)]+\zeta(t),
\end{aligned}
$$

for $\check{\varphi}(t) \simeq \varphi(t)$. The question is, how should one choose the estimate $\check{\varphi}(t)$ ?

One obvious possibility is to choose it from the immediately preceding photocurrent. equation (23) can be rearranged to get

$$
\varphi(t) \simeq\left[\check{\varphi}(t)+\frac{I(t)}{2 \alpha}\right]+\frac{\zeta(t)}{2 \alpha} .
$$

For a given $\check{\varphi}(t)$, one could thus form

$$
\check{\varphi}_{\mathrm{imm}}(t+\delta t)=\frac{1}{\delta t} \int_{t}^{t+\delta t}\left[\check{\varphi}(t)+\frac{I(s)}{2 \alpha}\right] d s .
$$

This has the MSE

$$
\begin{aligned}
\sigma_{\mathrm{imm}}^{2}(t+\delta t) & =\left\langle\left[\check{\varphi}_{\mathrm{imm}}(t+\delta t)-\varphi(t+\delta t)\right]^{2}\right\rangle \\
& \simeq \frac{1}{4 \alpha^{2} \delta t} .
\end{aligned}
$$

This diverges as $\delta t \rightarrow d t$, so clearly $\check{\varphi}_{\text {imm }}$ is not a good estimate. Instead, one needs a $\check{\varphi}(t)$ that involves a finite time average.

The optimal time-average for $\check{\varphi}(t)$ can be determined as follows. Say the MSE in $\check{\varphi}(t)$ is $\sigma^{2}(t)$. Over the interval $[t, t+\delta t)$, the diffusion of $\varphi(t)$ causes this to increase to

$$
\begin{aligned}
\sigma_{\text {old }}^{2}(t+\delta t) & =\left\langle[\check{\varphi}(t)-\varphi(t+\delta t)]^{2}\right\rangle \\
& =\left\langle[\check{\varphi}(t)-\varphi(t)]^{2}\right\rangle+\left\langle[\varphi(t)-\varphi(t+\delta t)]^{2}\right\rangle \\
& =\sigma^{2}(t)+\ell \delta t .
\end{aligned}
$$

By standard error analysis, the optimal $\check{\varphi}(t+\delta t)$ weights $\check{\varphi}(t)$ and $\check{\varphi}_{\text {imm }}(t+\delta t)$ appropriately:

$$
\check{\varphi}(t+\delta t)=\sigma^{2}(t+\delta t)\left[\frac{\check{\varphi}_{\mathrm{imm}}(t+\delta t)}{\sigma_{\mathrm{imm}}^{2}(t+\delta t)}+\frac{\check{\varphi}(t)}{\sigma_{\text {old }}^{2}(t+\delta t)}\right],
$$

where

$$
\frac{1}{\sigma^{2}(t+\delta t)}=\frac{1}{\sigma_{\text {imm }}^{2}(t+\delta t)}+\frac{1}{\sigma_{\text {old }}^{2}(t+\delta t)} .
$$

Taking $\delta t \rightarrow d t$ yields a differential equation for $\sigma^{2}(t)$ that has the stationary solution

$$
\sigma^{2}=1 / 2 \sqrt{N}
$$


which agrees with equation (21). Substituting this into equation (28) gives $d \check{\varphi}(t)=$ $\left(\ell / \sigma^{2}\right) \times(I(t) d t / 2 \alpha)$. Since the feedback sets $\Phi(t)=\check{\varphi}(t)+\pi / 2$, the feedback algorithm is simply

$$
\dot{\Phi}=\frac{\ell}{\sigma^{2}} \frac{I(t)}{2 \alpha} .
$$

Note that I did not labeled equation (21) the Heisenberg limit. That is because a phase-squeezed source can actually do better, with a scaling of $N^{-2 / 3}$, as derived in reference 40]. There is no point considering that here, because we are interested in the case where the beam to which one is locking arises from a laser. A phase-squeezed beam could only be produced by starting with a much more powerful source laser, and using a non-linear crystal for example. Such a process could be useful if we were interested in limiting the power in each individual beam, $f$. But here we are interested only in the ultimate limit set by the photon number in the source laser, $\mu$.

\subsection{Quantum limits to the laser as a clock}

We are now in a position to combine the results of the preceding two subsections. Using the Heisenberg limited laser linewidth (17), and the optimal (adaptive) result for phase locking (21) with $N=\kappa \mu / M \ell$, we obtain the HL

$$
(\delta \phi)_{\mathrm{HL}}^{2}=\sqrt{M} / 4 \mu,
$$

as quoted in equation (10). Using a standard (ideal) laser, and a non-adaptive technique, one would obtain the SQL

$$
(\delta \phi)_{\mathrm{SQL}}^{2}=\sqrt{M} / 2 \mu
$$

only a factor of two greater.

In practice, the laser linewidth $\ell$ is usually not limited by the uncertainty principle, and is far greater than $\kappa / 2 \mu$. The general expression, which may actually be of use to experimentalists, is

$$
(\delta \phi)^{2} \sim \sqrt{\frac{\hbar \omega M \ell}{P}} .
$$

For example, a one $\mathrm{mW}$ laser with a linewidth of $1 \mathrm{MHz}$ in the visible can synchronize the clocks of $M$ parties with a MSE of

$$
(\delta \phi)^{2} \sim 10^{-5} \sqrt{M}
$$

Thus, every person on the planet could use this laser independently to synchronize their clocks, with an error of order 1 radian (that is, of order $10^{-15} \mathrm{~s}$ ).

Of course, this would not be the most efficient way to establish an optical-scale time standard among the world's population. Rather, if the above, very ordinary, laser had to be used as the time standard, then it would be best to lock it to another laser, with far better properties (higher power, lower linewidth) and then to use that laser as the new standard. Providing the second laser was good enough, this would result in no further increase in mean square phase error, relative to the original source laser, 
above the $10^{-5}$ radians $^{2}$ between the source and second laser. This reinforces the idea that the phase reference supplied by the source laser is not a quantum channel. If used wisely, the information can be shared amongst many parties without further loss. Thus it makes sense to think of it as classical information, not a quantum resource.

\section{Conclusion}

I have argued that, even though it is correct to represent the quantum state of a laser by a mixture of coherent states (1) or number states (2), the output of a laser is truly coherent in any meaningful sense of the words. In particular, it is also quite correct to represent the state as a single coherent state $|\sqrt{\mu}\rangle$. That is because the state of any oscillator, except for an energy eigenstate, can only be defined relative to some time reference. If a laser itself is used as the time reference, then by definition it is coherent relative to itself, with phase zero. Thus, the idea of Rudolph and Sanders, accepted also by van Enk and Fuchs, that there is some 'true coherence' which a laser lacks (as yet) is illusory. Thus there is no sound basis for the former's criticism of the continuous variable quantum teleportation experiment [10].

In defending this view I have made a number of important points. First, absolute phase is meaningless (unless one attributes a special status to human consciousness, but even this cannot experience anything on a time scale much less than $\sim 10^{-1} \mathrm{~s}$ ). There is only relative phase, and a laser is as good a phase reference as any other. Indeed, a laser deserves to be called a clock because its time standard is fungible with any other clock. The same is not true of a Bose condensate, for example.

Second, even though equation (2) implies that a sufficiently clever observer could find out which number state a laser is in, this does not contradict my statement that a laser used as a clock is in a coherent state. That is because finding out which number state a laser occupies would destroy the phase information necessary for it to be used as a clock. A quantum state can be a state of purpose as well as a state of knowledge.

Third, the existence of optical phase cannot be denied on the basis of a superselection rule for energy, because no such super-selection rule exists. I have shown this by carefully explaining that a super-selection rule relates to a conserved quantity if and only if it is of the form of a direct sums of other quantities, and that the rule applies to these other, non-conserved quantities. Energy is never of this form.

Fourth, it is not necessary to have a channel which can transmit quantum information in order to establish a time reference, using a laser or any other means. Clock synchronization in the teleportation experiment can thus be regarded as quite separate from the quantum teleportation.

Fifth, although a given laser can only synchronize several parties to a finite degree of accuracy, this is not an argument against it being a clock. That argument would be true of any clock with a finite excitation. In this paper I have determined, to my knowledge for the first time, the quantum limits to clock synchronization using a laser. The (non-obvious) result is that the accuracy with which the phase of $M$ parties can 
be independently locked to that of a continuously running laser containing on average $\mu$ quanta of excitation is limited by

$$
(\delta \phi)^{2} \geq \sqrt{M} / 4 \mu .
$$

None of my arguments strictly disprove the existence of absolute time, even if it is unobservable. So, if one wished, one could insist that the laser should always be described by equations (1) or (2) rather than a single coherent state. One could follow Rudolph and Sanders and insist therefore that CVQT has not been demonstrated. But one should be aware that by doing this one must also insist that there would never be any states with well-defined phase to be teleported either. And that no experiment has ever demonstrated optical squeezing, let alone CV optical entanglement. In fact, if

one were to follow the arguments of RS to their logical conclusion, one would have to discount all experiments using a "mere clock", rather than an absolute time standard. To practising scientists and engineers, this would be a ludicrous position.

\section{Acknowledgments}

I gratefully acknowledge friendly discussions with S. Bartlett, S. Braunstein, S. van Enk, C. Fuchs, J. Gea-Banacloche, T. Rudolph, B. Sanders, and R. Spekkens.

[1] T. Rudolph and B. C. Sanders, Phys. Rev. Lett. 87, 077903 (2001).

[2] H. M. Wiseman, quant-ph/0104004 (2001).

[3] S. J. van Enk and C. A.Fuchs, Phys. Rev. Lett. 88, 027902 (2002).

[4] S. J. van Enk and C. A.Fuchs, Quantum Information and Computation 2, 151 (2002).

[5] T. Rudolph and B. C. Sanders, quant-ph/0112020 (2001).

[6] K. Nemoto and S. L. Braunstein, quant-ph/0207135 (2002).

[7] H. M. Wiseman, J. Mod. Opt. 50, 1797 (2003).

[8] H. Heydari and G. Björk, quant-ph/0302150 (2003).

[9] B. C. Sanders, S. D. Bartlett, T. Rudolph, P. L. Knight, Phys. Rev. A 68, 042329 (2003)

[10] A. Furusawa et al., Science 282, 706 (1998)

[11] M. Sargent, M. O. Scully, and W. E. Lamb, Laser Physics (Addison-Wesley, Reading Mass., 1974)

[12] D. F. Walls and G. J. Milburn, Quantum Optics (Springer, Berlin, 1994).

[13] K. Mølmer, Phys. Rev. A 55, 3195 (1996).

[14] P. Kok and S. L.Braunstein, Phys. Rev. A 61, 042304 (2000).

[15] T. Rudolph, pers. comm. (2002).

[16] H. A. Haus, Electromagnetic Noise and Quantum Optical Measurements (Springer, Berlin, 2000).

[17] L. H. Ryder, Quantum Field Theory (Cambridge: Cambridge University Press), pp. 115-26 (1985).

[18] H. M. Wiseman, Phys. Rev. A 56, 2068 (1997).

[19] D. T. Pegg, J. Phys. A 24, 3031 (1991).

[20] R. Penrose, The Emperor's New Mind (London: Vintage), pp. 439-44. (1990).

[21] S. J. Jones et al., Science 288, 635 (2000).

[22] C. A. Fuchs, pers. comm. (2002).

[23] This is ironic since they state that they "model the laser as a one-sided cavity driven by a constant force (a voltage or an external field)", an unphysical model which would produce a state of definite phase!

[24] C. M. Caves, C. A. Fuchs, and R. Schack, Phys. Rev. A 65, 022305 (2002).

[25] E. Schrödinger, Proc. Cambridge Philos. Soc. 32, 446 (1936).

[26] M. Vujičić and F. Herbut, J. Phys. A 4 21, 2931 (1988). 
[27] S. D. Bartlett and H. M. Wiseman, Phys. Rev. Lett. 91, 097903 (2003)

[28] H. Barnum, M. A. Nielsen, and B. Schumacher Phys. Rev. A 57, 4153 (1998).

[29] M. A. Nielsen, E. Knill, R. Laflamme, Nature 396, 52 (1998)

[30] H. M. Wiseman, Phys. Rev. A 60, 4083 (1999).

[31] I. L. Egusquiza, L. J. Garay, and J. M. Raya, Phys. Rev. A 59, 3236 (1999).

[32] S. A. Diddams et al, Science 293, 825 (2001).

[33] S. L. Braunstein et al., Phys. Rev. Lett. 86, 4938 (2001).

[34] A. L. Schawlow and C. H. Townes, Phys. Rev. 112, 1940 (1958).

[35] W. H. Louisell, Quantum Statistical Properties of Radiation (John Wiley \& Sons, New York, 1973).

[36] R. Loudon, The Quantum Theory of Light (Oxford University Press, Oxford, 1983).

[37] S. M. Barnett, S. Stenholm, and D. T. Pegg, Opt. Comm. 73, 314 (1989).

[38] M. Hillery, M. Freyberger, and W. Schleich, Phys. Rev. A 51, 1792 (1995).

[39] Y. Yamamoto and H. Haus, Phys. Rev. A 41, 5164 (1990).

[40] D. W. Berry and H. M. Wiseman, Phys. Rev. A 65, 043803 (2002).

[41] H.M. Wiseman, Australian optical Society News 16 No.2, p.14 (2002), quant-ph/0206124

[42] M.A. Armen et al, Phys. Rev. Lett. 89, 133602 (2002).

[43] H.M. Wiseman and R.B. Killip, Phys. Rev. A 56, 944 (1997). 